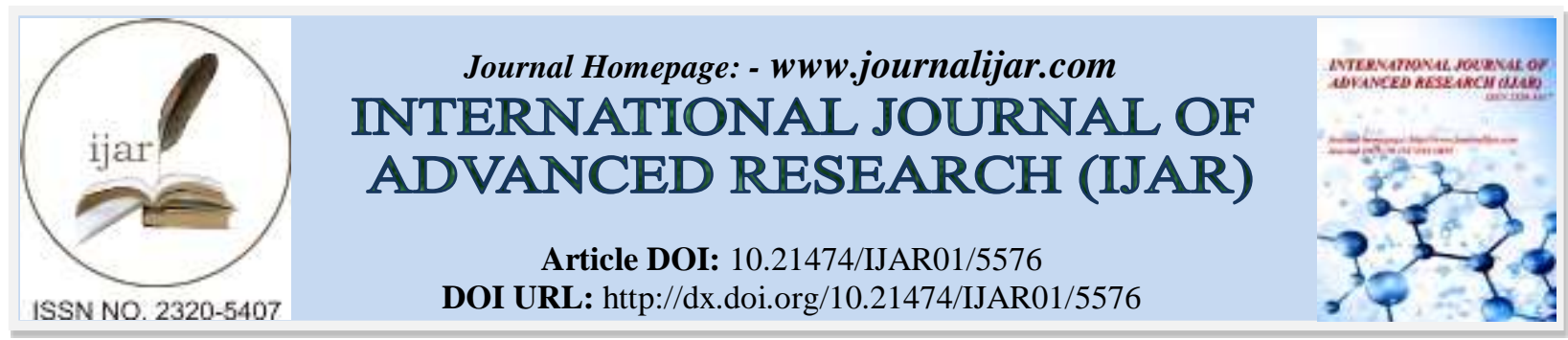

RESEARCH ARTICLE

\title{
FIBRONECTIN EXPRESSION PATTERN IN SKELETAL MUSCLE REGENERATION.
}

${ }^{*}$ Taghreed Abdul Rasool Ali ${ }^{1}$ and May Fadhil Al- Habib".

1. Department of histology \& Embryology, University of AL- Nahrian, Baghdad, Iraq.

2. Department of histology \& Embryology, University AL- Nahrian, Baghdad, Iraq.

\section{Manuscript Info}

Manuscript History

Received: 09 August 2017

Final Accepted: 11 September 2017

Published: October 2017

Key words:-

Skeletal muscle, regeneration,

fibronectin, expression.

\section{Abstract}

Skeletal muscle is the largest component in the humane bodies, the main function of skeletal muscle is drive locomotion. Muscle fibers composed of myofibrils, the myofibrils are composed of actin and myosin filaments interact to from actinomyosin cross bridge, fibronectin is a high-molecular weight glycoprotein of the extracellular matrix that binds to membrane-spanning receptor proteins called integrins .Similar to integrins, fibronectin binds extracellular matrix components such as collagen, fibrin, and heparin sulfate proteoglycans. The present study enrolled 40 mature male rabbits. The Extensor digitorum longus muscle was set as amodel for skeletal injury, extensor digitorum longus muscle taken after $3 \& 6$ weeks post operatively. Muscle tissues were histologically prepared for paraffin section a proceeded with immunohistochemical reaction fibronectin $\mathrm{Ab}$. The Aperio software for quantitative immunohistochemical reaction was used. Fibronectin antibody was used in this study to asses it is role and changes during the process of regeneration there was a significant difference between the $3^{\text {rd }}$ week's groups and other 2 groups $\left(6^{\text {th }}\right.$ weeks and control groups).

This study occluded that there is an important role for fibronectin in skeletal muscle regeneration

Copy Right, IJAR, 2017,. All rights reserved.

\section{Introduction:-}

Skeletal muscle is the largest component in the humane bodies that comprise about $40 \%$ of total body mass, the main function of skeletal muscle is drive locomotion. Muscle fibers composed of myofibrils, the myofibrils are composed of actin and myosin filaments interact to from actinomyosin cross bridge, while generate muscle contraction, myosin and actin filaments are organized into sarcomeres which are the functional units of muscle contraction, the sarcomere is responsible for the striated appearance of skeletal muscle and form the basic machinery necessary for muscle contraction (1). 
It, has the ability to regenerate new muscle fibers after it has been damaged by injury or as a consequence of diseases such as muscular dystrophy Muscle fibers are syncytial cells that contain several hundred nuclei within a continuous cytoplasm. Therefore, whether the process of regeneration depends upon the fusion of mononucleotide precursor cells or upon the fragmentation of dying muscle fibers, which release new cells(2).

Muscle regeneration recapitulates many aspect of embryonic myogensis and is an important homeostatic process of the adult skeletal muscle, which after development, retains the capacity to regenerate in response to appropriate stimuli, "qualified" environment is necessary to guarantee and achieve functional result, therefore loss of control over muscle precursor cell these cell fate decisions could lead to a pathological trans differentiation leading to pathologic defect in the regenerative process( 3 ).

During healing of injured muscle two process are competing the regeneration of disruptive muscle and the degeneration at the site of injury which may inhibit the complete regeneration of muscle tissue by excessive formation of granulation tissue(4).

Skeletal muscle has an excellent ability to regenerate. The satellite cell (muscle precursor cell) which lie beneath the basement membrane are activated in response to injury. Activated muscle satellite cell (myoblast) proliferate, differentiation and fuse in to myotube, mature to form myofibers, (5).

Fibronectin is high - molecular weight cell surface associated glycoprotein present in most tissues and plasma, because of the high affinity of fibronectin to collagen, it is co- distributed with collagen in connective tissue, mesenchyme, and the basement membrane. Fibronectin plays an important role in various cellular activities, such as cell to cell interaction, cell -to - substratum (mostly collagen) binding, and cell spreading and migration (6).

\section{Materials and methods:-}

\section{Animals housing \& feeding:-}

The present study was done on 40 mature male rabbits. The animals were placed in to plastic cages made off with standardized criteria like: non-toxic material, non- pours, round corners, ease to clean. Food \& tap water was freely offered. Each cage contained two animals.

The cages were kept at $25^{\circ} \pm 2 \mathrm{C}$ of room temperature in a clean and well ventilated room.

\section{Surgical operations:-}

1- The operations were done under general anesthesia by using IM injection of ketamine \& xylazine. The doses of the calculated dose $=$ measured dose $\mathrm{x}$ weight $/$ concentration. Measured dose of ketamine for rabbit is $35 \mathrm{mg} / \mathrm{kg}$.

Measured dose of xylazine for rabbit is $5 \mathrm{mg} / \mathrm{kg}$.

4- The Extensor digitorum longus muscle was cut transverse with separation of its blood supply then immediately replaced in its position and sutured by using Horizontal matters suture pattern with an absorbable suture material.

5 the subcutaneous and skin above was stand

6- An open splint was placed on the wound area to ensure that the movement of the hind limb is minimized. The splint contained a window to monitor the development of wound healing and contamination.

7- the animal were kept under Antibiotic cover ceftriaxone was given for 3 day post operationally to each animal and then samples taken after $3 \& 6$ weeks of operation . 


\section{Sampling and grouping:-}

Normal skeletal muscle were taken from EDLM of rabbits that did not went under muscle injury, they act as control group. In regards to operated animals they were divided into 2 experimental group $3^{\text {rd week }}$ post-operative group and $6^{\text {th }}$ week post-operative group each enrolled is animal

\section{Fibronectin staining:}

- $\quad$ Tissue and amply were prepared for paraffin block sectioned at 4 micron

- 4 micron thick tissue place on positively changed slide sections were deparaffinized as follows: oven for 15 minutes, xylenes for 5 minutes each, $100 \%$ ethanol for 10 minutes, $90 \%$ ethanol for 5 minutes and $70 \%$ ethanol for 5 minutes. Then they were washed in distal water for 5 minutes on a stir plate than wash by PBS.

- All subsequent steps were carried out at room temperature in a humidified chamber, were allowed to reaching room temperature prior to use. Tissue sections should not be allowed to dry out at any time during the procedure

- Then put enzyme pepsin ( $1 \mathrm{ml}$ dilution by $2 \mathrm{ml}$ of distal water) on tissue and place in incubator for 10 minute, degree $37 \mathrm{c}$ then wash by PBS

- Incubate slides in hydrogen peroxide for 5 minutes, and wash by PBS.

- Apply blocking regent and incubate for 5 minutes, and wash by PBS.

- $\quad$ Apply primary antibody and incubated for 24 hours ,then washed by PBS

- Then apply house reddish peroxide (HRP) and incubated for 20 minutes, washed by PBS.

- add 0.3 milimicrone of DAB chromogen with 100 milimicrone of DAB substrate and apply on tissue for 10 minutes then wash by PBS

\section{Result:-}

The anti-fibronectin $\mathrm{Ab}$ was used to demonstrate the presence of fibronectin between skeletal muscle fibers and to quantify the differences of activity and the role of it in skeletal muscle regeneration. The fibronectin distribution in both experimental groups and control showed multiple differences, the fibronectin usually present in the all types of connective tissue surrounding muscles (epimysium, perimysium and endomysium). In control groups, the fibronectinre activity in the endomysium showed a very thin layer which corresponded the basement membrane of muscle fibers, while the epimysium and perimysium showed a heavy reactive stainability towards fibronectin antibody, (figure 1)

The expression of fibronectin $\mathrm{Ab}$ was seen mainly in between the skeletal muscle fibers and the staining reactivity was not observed insidemuscle fiber or their nuclei. The nuclei component stained with counter stain -Harris Hematoxylin and seen in (Figure 2).

In regenerated muscles 2 different post-operative timing groups were investigated using antibody( NBP1-51723), $3^{\text {rd }}$ post-operative and $6^{\text {th }}$ post-operative respectively and as it was mention above the process of regeneration and degeneration is dynamic one. the changes seen in the $3^{\text {rd }}$ weeks post operatively were striking, multiple profile was seen in the $3^{\text {rd }}$ Post-operative groups, at the same time when the degeneration process was going on at the site of injury of muscle graft was found that with present of mononuclear infiltration cells and heavy vascularity a new muscle cells at different level of maturity of also seen raging between new muscles, type $2 \mathrm{C}$ fibers and myotube, (Figure 3).

A striking feature that was noticed in the $3^{\text {rd }}$ post-operative week group was the presence of intense reactivity of fibronectin $\mathrm{Ab}$ inside the sarcoplasm of newly formed cells unlike the picture seen in control group where the sarcoplasm showed negative reaction toward fibronectin Ab, (Figure 4, 5, 6). 
Peripheral myofibers that survived injury remains intact forming a zone surrounding the injured area were the muscle sarcoplasm devoid of fibronectin reaction, (Figure 7).

The newly formed muscle fibers showed no clear endomysium reactivity towards fibronectin while showed heavy reactivity inside of sarcoplasm, the being dynamic process mature muscle fibers was seen at the end of $3^{\text {rd }}$ postoperativealthough they were a very few but this muscle mature muscle fibers showed a clear cytoplasm with negative reactivity toward fiber only a thin layer of endomysium surrounding the peripheral of sarcoplasm, (Figure 8).

Regenerative process was completed by the end of $6^{\text {th }}$ post-operative week, the entire muscle showed organized regeneration muscle fibers arranged in bundles with all histological feature of maturity. The fibronectin reaction was limited to a thin strand of reaction in between mature fibers, while the sarcoplasm was devoid of any reaction, (Figure 9)

The overall appearance the regenerated of skeletal muscle was similar to the normal non injured muscle apart from the presence of larger area of connective tissue in regenerated muscle than in control groups, the intensity of fibronectin in connective tissue was heavy (Figure 10).

Not all injury muscle ended in a successful regeneration, some ended in failure of regeneration and formation of heavy granulation tissue scar, still although failure is seen small foci of new muscle cells appeared between granulation tissue (Figure 11).

The degree of positivity measured by using aperio program and this program identified different color in each section according to the intensity of the reaction

* The strong positively appear in a brown color.

* The positively appear in orange color.

* The weak positively appear in yellow color.

* The negative appear in blue color.

- The white color indicate no tissue in the sections.

The statistical analysis of fibronectin results showing different significance between normal group, $3^{\text {rd }}$ postoperative groups and $6^{\text {th }}$ post-operative groups within on-significant between difference normal groups and $6^{\text {th }}$ postoperative groups table, (1) and figure (12). 


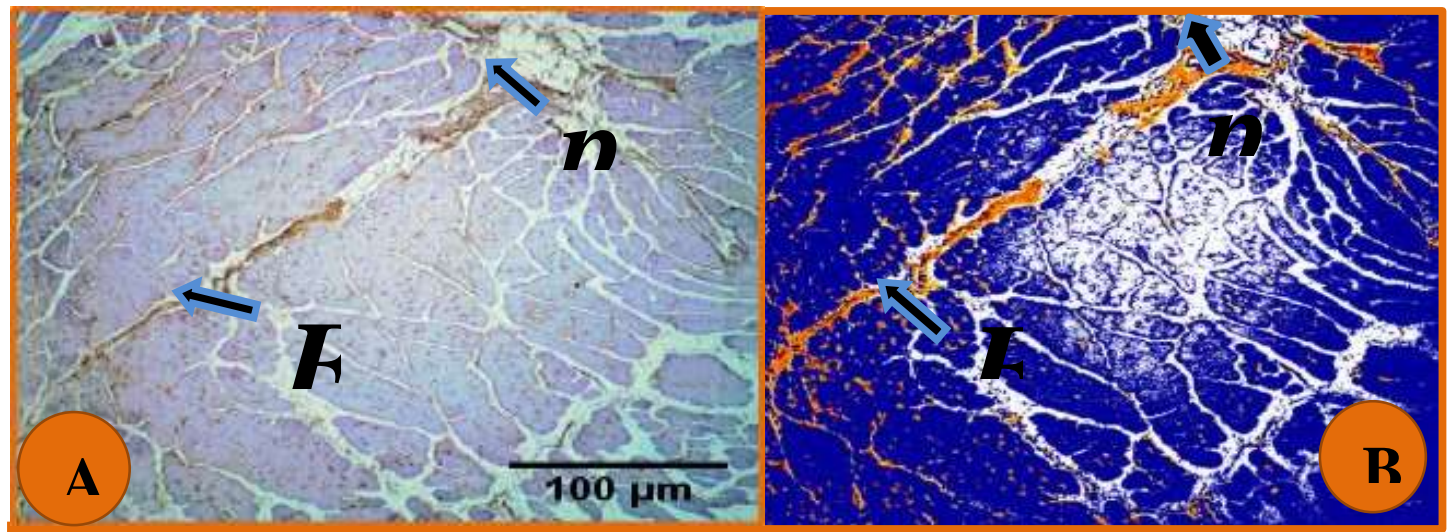

Fig: (1) Cross sections in control group showing A: faint distribution of fibronectin at the endomysium (E) \& heavy reactivity in perimysium \& around blood vessels B: the same image analyzed using the Aperio softwear fibronectin positive reactivity ranging between yellow to brown color .(Fibronectin Ab, control group, X10)

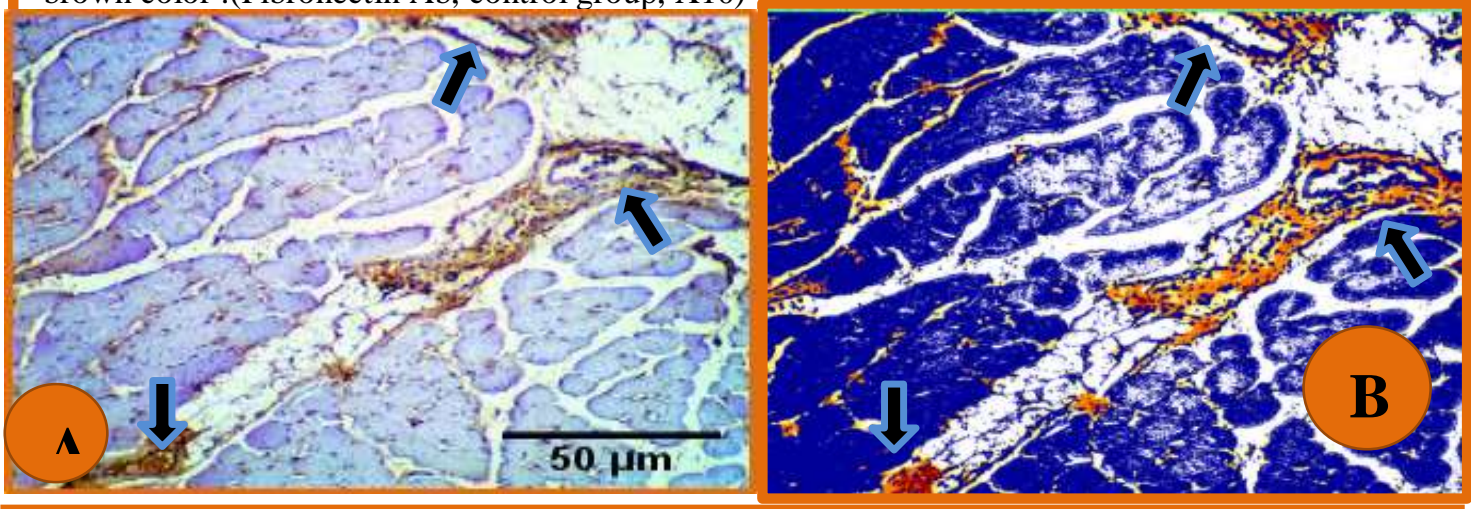

Fig: (2) Cross sections in the skeletal muscle showing A : sarcoplasm of muscle fiber free of any reactivity toward fibronectin $\mathrm{Ab}$ with intense reaction around blood vessels in the perimysium ( $\Rightarrow$ ) B: the same image using Aprio showing positive(yellow -brown )and negative blue reaction. (Fibronectin $\mathrm{Ab}$, control group, X20)

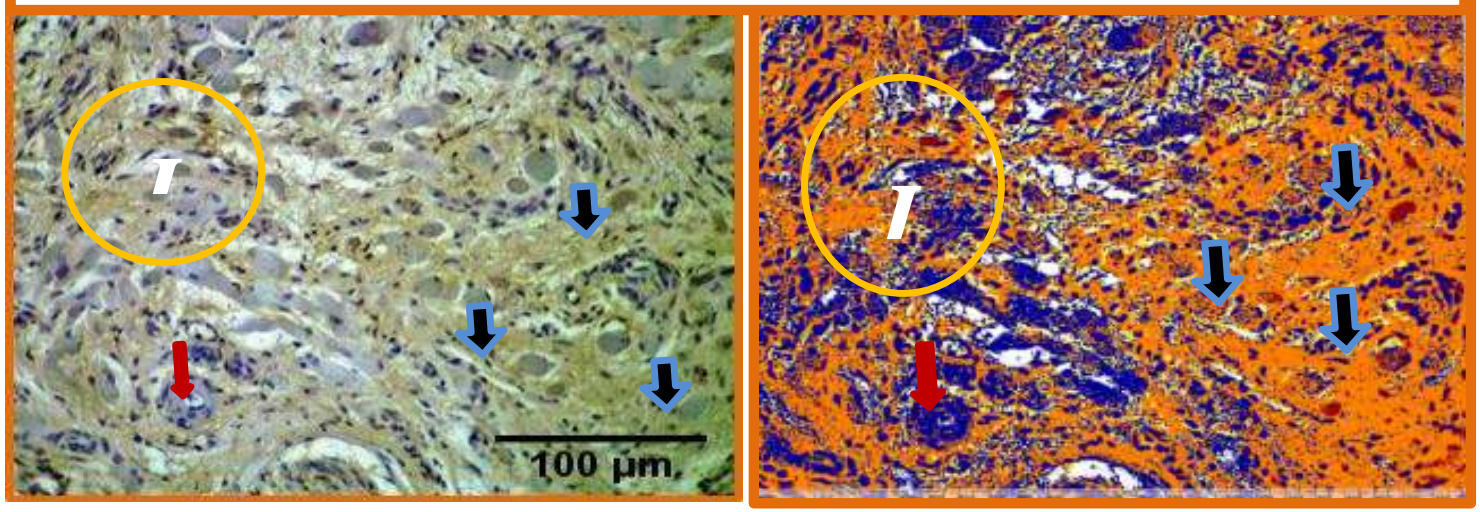

(Fig:3) A: Cross sections in the skeletal muscle regeneration showing inflammatory cells (I) $\overrightarrow{, \vec{m}}$ muscle fibers (_) with a heavy constituent of connective tissue element and large number of blood vessels $(\longrightarrow$ ) B: same image seen with Aperio softwear showing the same feature with emphasis on the appearance of fibronectin reactivity in new muscle sarcoplasm . 

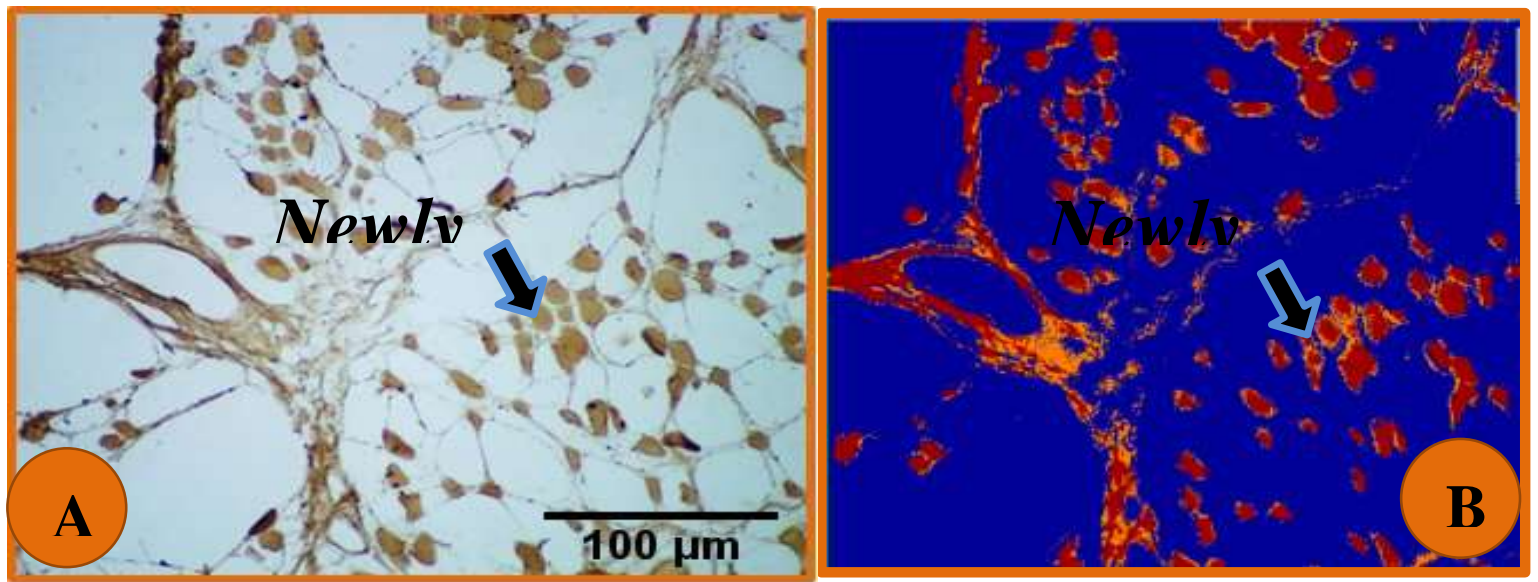

(Fig: 4)A: section in regenerating skeletal muscle at higher magnification showing the intense reactivity at the sarcoplasm of newly formed muscle together with the perimysium B: same image with positive ( brown )(Fibronectin Ab, $3^{\text {rd }}$ week , X20)

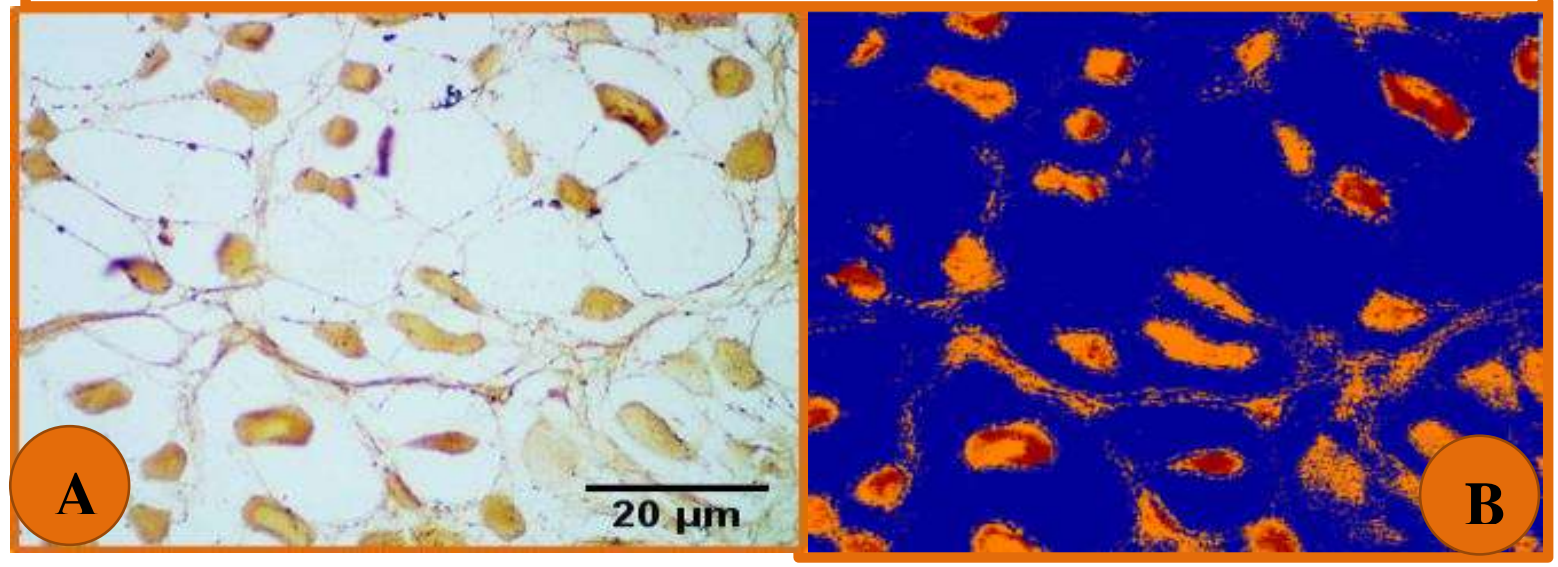

(Fig: 5)A: section in the skeletal muscle regeneration showing newly formed muscle with intense reactivity toward fibronectin \&extracellular matrix weak reactivity B: the same image using Aperio reactivity of fibronectin appear as organ - brown area .(Fibronectin Ab. $3^{\text {rd }}$ week X 40).

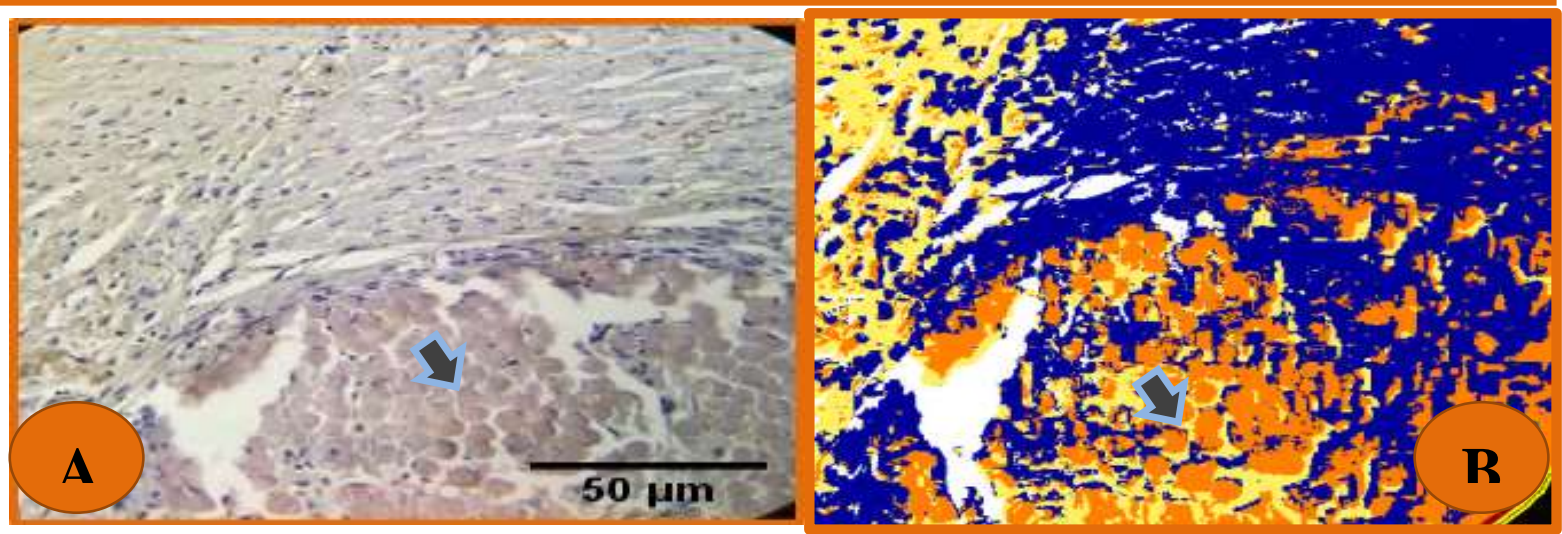

(Fig: 6) A: Cross sections in the skeletal muscle regeneration showing focus of regeneration at the site of injury surrounded by mature muscle fibers notice sarcoplasmic reactivity in new muscle B: the Same image using Aperio showing distribution of fibronectin represent by brown -orange color indicative intense reactivity. (Fibronectin $\mathrm{Ab}, 3^{\text {rd }}$ week, X20). 

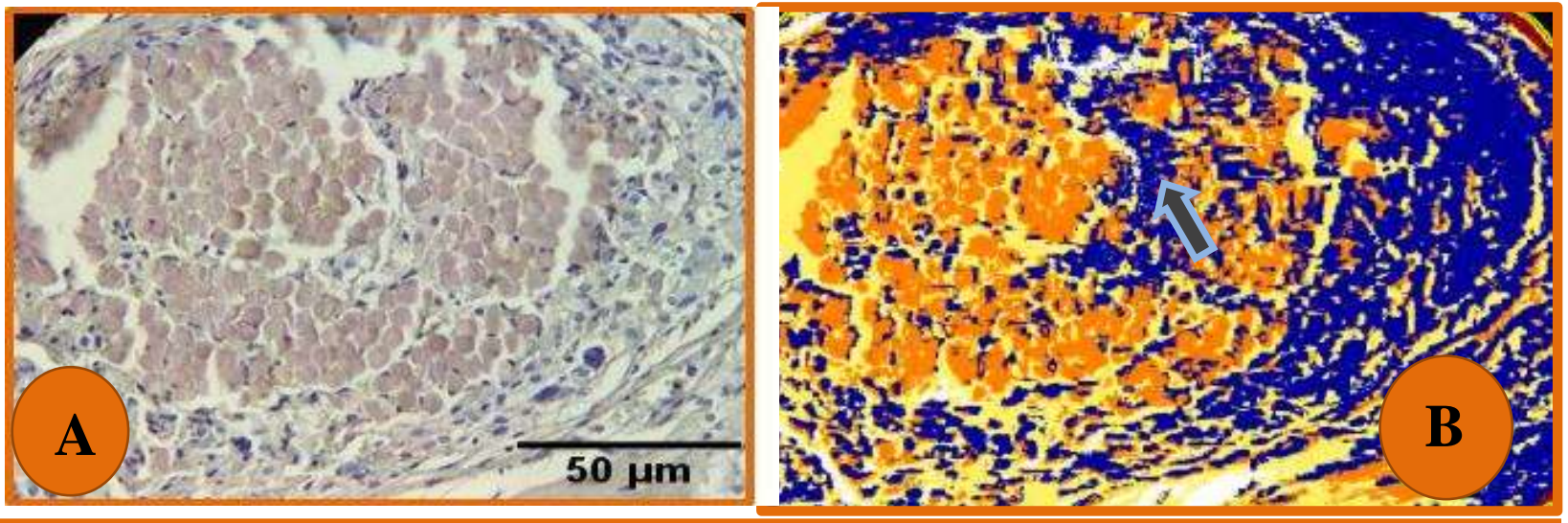

(Fig: 7) A: Cross sections in the skeletal muscle regeneration showing focus of regeneration at the site of injury surrounded by mature muscle fibers, notice sarcoplasmic reactivity in new muscle B: the Same image using Aperio showing distribution of fibronectin represent by brown -orange color indicative intense reactivity (Fibronectin $\mathrm{Ab}, 3^{\text {rd }}$ week, X20).

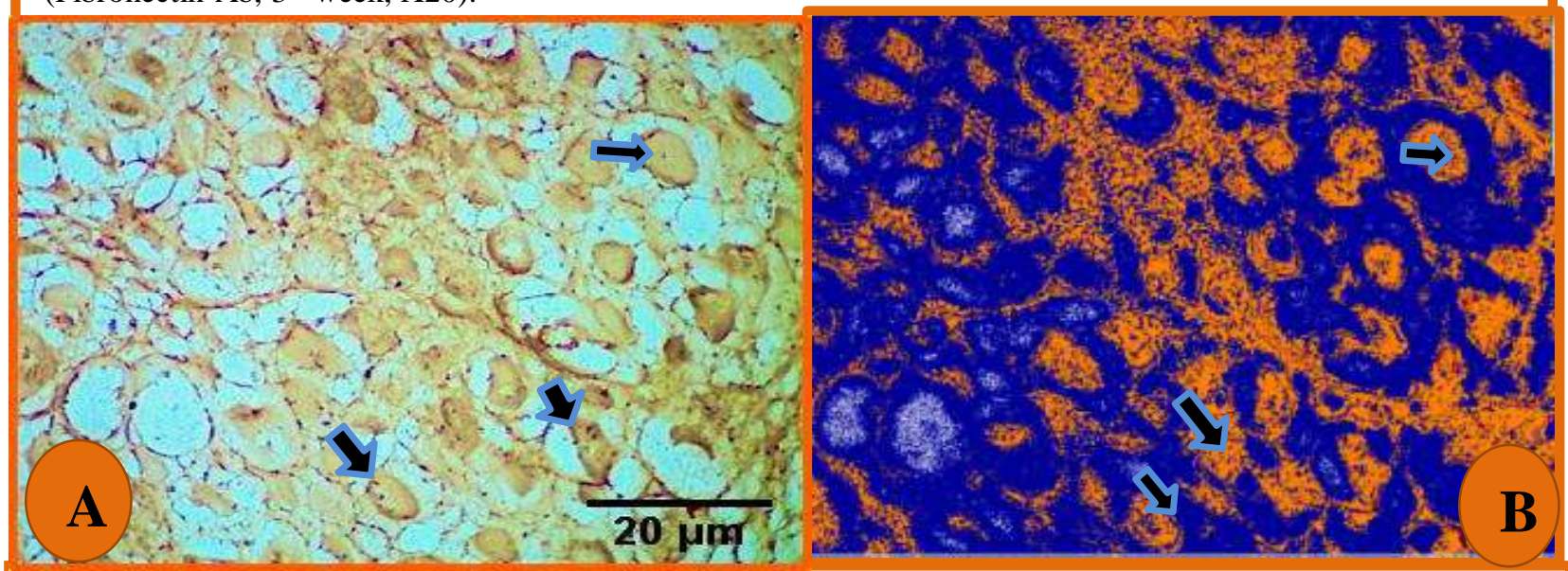

(Fig:8) A: Cross sections in the regenerating skeletal muscle fibers showing the appearance of mature muscle at the same time of the presence of non-mature cell, mature cells cell sarcoplasm devoided of any reactivity toward fibronectin $\mathrm{Ab} \mathbf{B}$ : same image showing negative Fibronectin reactivity in mature cell sarcoplas ( Fibronectin Ab, 3rd week, X 20).

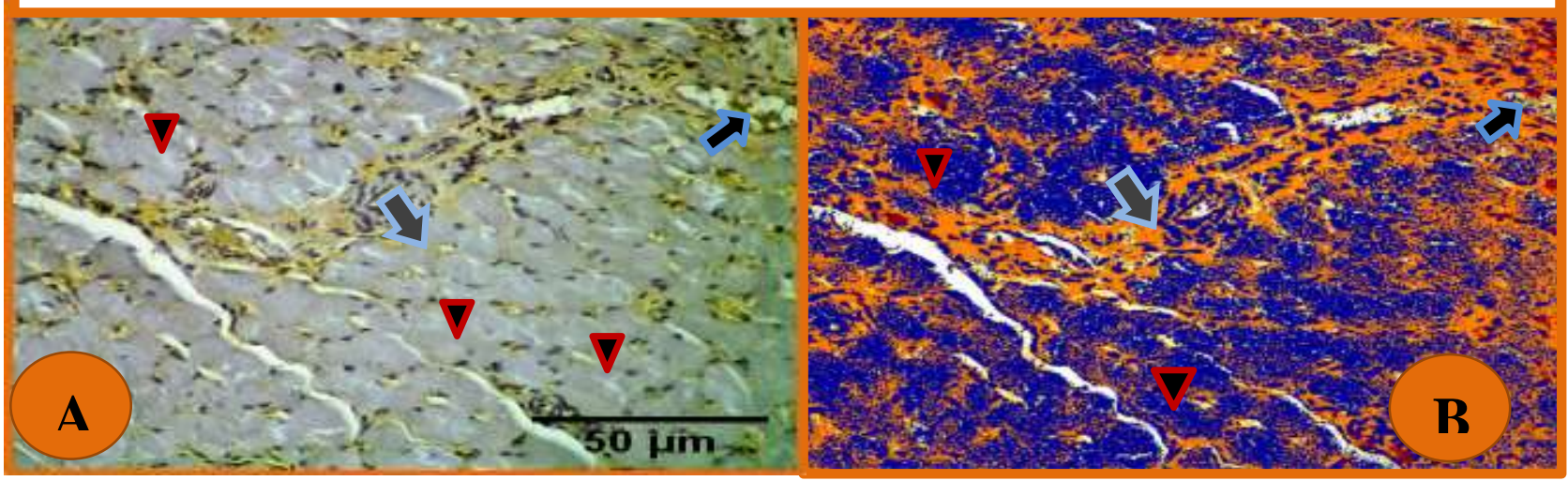

(Fig: 9)A: Cross sections in the skeletal muscle regeneration showing mature muscle fiber ( $\boldsymbol{\nabla}$ ) with no sarcoplasm reactivity , the reaction was confined to extracellular matrix and round blood vessels

$(\Rightarrow$ )B: Same image analysis by Aperio when sarcoplasm showed negative blue color. (Fibronectin Ab, $6^{\text {th }}$ week group, X20). 

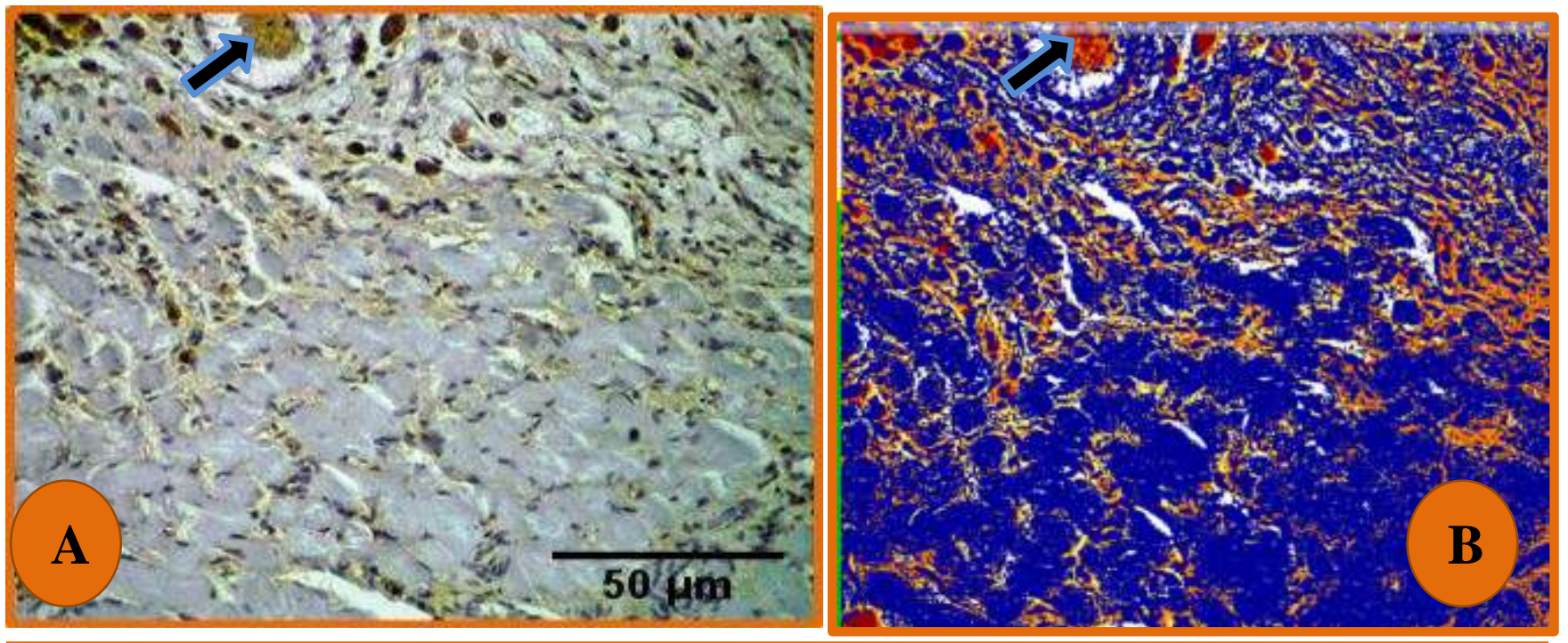

Fig: (11) A: Cross sections in regenerated muscle showing mature muscle fibers after injury with reaction toward fibronectin $\mathrm{Ab}$ confined to conection tissue, still very few non mature muscle fiber can be seen $(\rightarrow)$ B: same image showing negative Fibronectin reactivity in mature cell sarcoplasm. (Fibronectin Ab, $6^{\text {th }}$ week group, X20).

Table (1):- Fibronectin expression in different experimental groups

\begin{tabular}{|l|l|l|}
\hline The Groups & Mean \pm SE (Pixel /Micron) & P-value \\
\hline $\begin{array}{l}\text { Fibronectin expression in control } \\
\text { group }\end{array}$ & $0.157 \pm 0.01 \mathrm{~B}$ & 0.0373 \\
\hline $\begin{array}{l}\text { Fibronectin expression in } 3^{\text {rd }} \\
\text { week group }\end{array}$ & $0.497 \pm 0.02 \mathrm{~A}$ & \\
\hline $\begin{array}{l}\text { Fibronectin expression in } 6^{\text {th }} \\
\text { week group }\end{array}$ & $0.163 \pm 0.01 \mathrm{~B}$ & \\
\hline $\begin{array}{l}\text { (P<0.05). } \\
\text { Means having with the different letters in same column } \\
\text { differed significantly. }\end{array}$ & \\
\hline
\end{tabular}




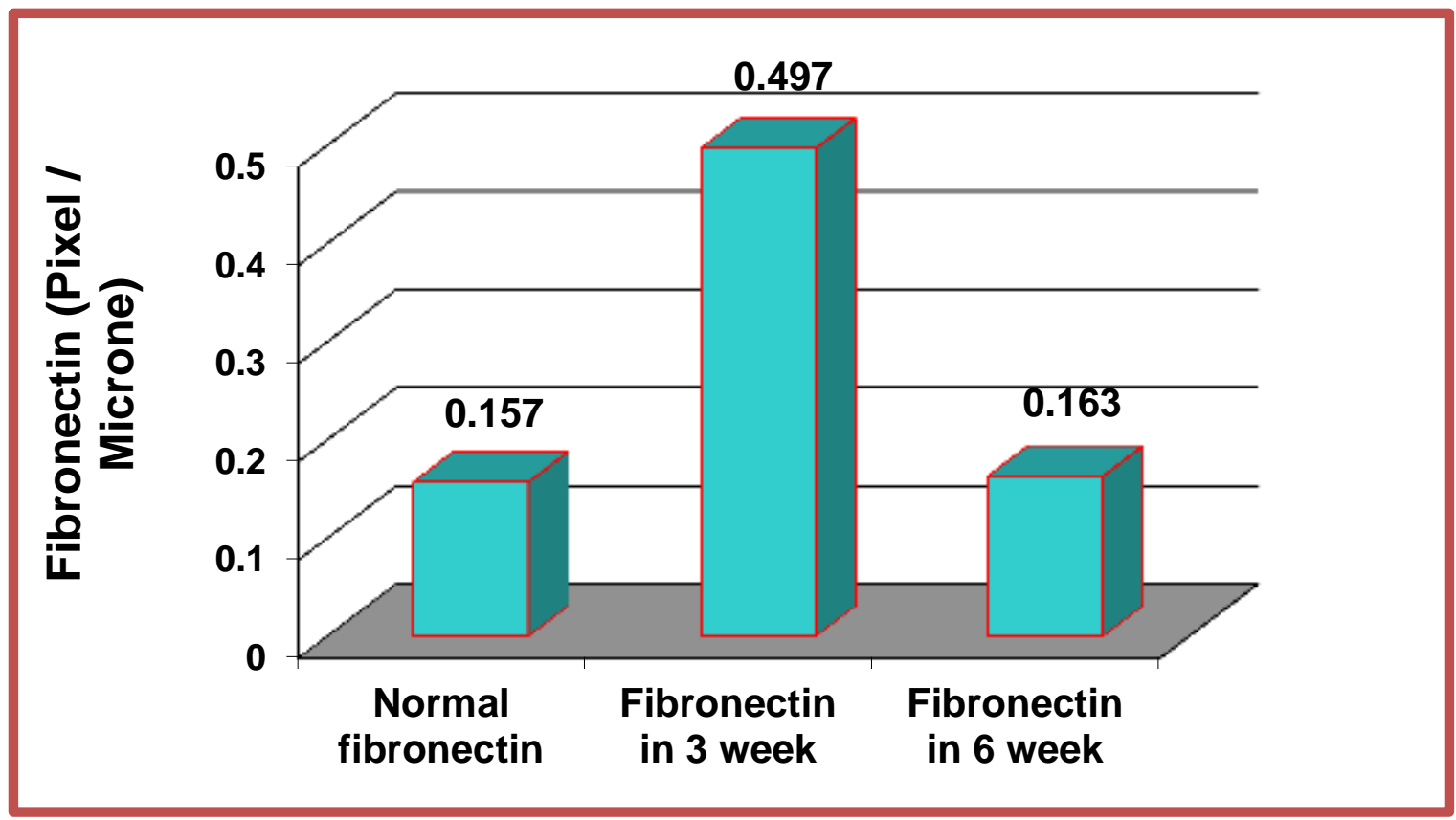

Figure (12)-: compare between different groups in fibronectin expression during skeletal muscle regeneration

\section{Discussion:-}

Fibronectin is high molecular glycoprotein associated with different surface of different type of cell, found in most tissue and plasma, fibronectin plays and important role in different cellular activates. Fibronectin plays important role in different cellular activates such as cell to cell contact binding cell separated, migration and differentiation, fibronectin is also closely related to process of cellular development and tissue remolding most studies describe a role of fibronectin in the vitro differentiation $(7,8)$.

However it is function has not been studied thoroughly and it is poorly understood. This study attempt to investigate possible changes in the expression of fibronectin during experimental skeletal muscle regeneration, to understand the important of fibronectin immediately enter action with extracellular matrix changes during muscle regeneration. A comparative study was set to assess this changes and it found that the active stages of regeneration represent by $3^{\text {rd }}$ weeks post-operative groups have a highly intense expression of fibronectin.

The distribution of fibronectin was investigated by (Raddi, 1998) during the stages ischemia, degeneration and regeneration after injury. He recorded that there is gradual loss of endomysium through the stages of ischemia and degeneration and spreading of fibronectin inside the sarcoplasm at early regeneration stage was established. Thin finding correspond agree with our finding regarding the elevation of fibronectin expression in $3^{\text {rd }}$ week postoperative week group, (9)

The exact mechanism of endomysia fibronectin depletion and disappearance is not recognized yet, however numerous explain suggestion can be mad to explain this one solid base of explanation is the increase enzymatic cleavage and degradation, (10)

All the myofibers possessed fibronectin as a thin, continuous layer in the endomysium . Epimysium, and perimysium as well, contained fibronectin and the sarcoplasm of the normal muscle fibers, however, was devoid of fibronectin also blood vessels and capillaries heavily stained with fibronectin, especially in the region corresponding to the basement membrane of the endothelium, previous studies agree with our results on presence of fibronectin in normal muscle tissue and were reported that in normal muscle tissue, fibronectin was located in the pericellular (endomysium) region of the myofiber and absent in the sarcoplasm. The nerves and blood vessels present within the 
muscle also displayed fibronectin, the distribution of fibronectin in normal muscle, nerve, blood vessels, and capillaries was in agreement with previous reports,(11)

A previous study on expression of laminin, fibronectin and tenascin-C in skeletal muscle of dystrophic mice demonstrated that in mature skeletal muscle, foci of regenerating tissue characterized by small-diameter muscle fibers, expanded interstitium, and infiltrating mononuclear cells are evident and characterized by distinct expression patterns of adhesion and ECM molecules. Most noteworthy were the expression of fibronectin, tenascin-C, and laminin were upregulated in the foci of regenerating tissue in mice skeletal muscle. Comparison with the expression pattern of an endothelial cell marker demonstrated that the increased expression of VCAM-1 and ICAM-1 in the focal lesions was probably largely due to increased vascularization of the regenerating muscle tissue.

Changes in fibronectin distribution during myogenesis in vitro have been described. The presence of a diffuse fibrillary network of fibronectin was observed on the cell surface of the myoblasts and it disappeared from the cell surface during and after myoblast fusion. These results proposed that fibronectin may act as a barrier to myoblast fusion, and its disappearance at certain stages of development results in myoblast fusion and myogenesis. The changes in its expression can be used to follow the fate of the basement membrane during regeneration of skeletal muscle. The fact that fibronectin disappeared from the endomysium of degenerating myofibers and appeared again as a prominent ring around the newly regenerated myotubes, means that the original basement membrane or its fibronectin component was lost and a new basement membrane component(s) formed around the myotubes. This finding indicates that the basement membrane is not an inert structure, as described in previous electron microscope studies, and components of it change quite rapidly during myofiber degeneration. It is known that the basal lamina is important during muscle regeneration and persists; within it the myoblasts and myotubes originate and mature,(12)

The present study showed that at the $3 \mathrm{rd}$ and $6^{\text {th }}$ weeks of muscle regeneration process the entire muscle was filled with original and regenerated muscle fibers of various sizes in addition to that thin strands of fibronectin were seen within the sarcoplasm of large regenerated myofibers. The myofibers became enlarged by 6 week the entire muscle was filled with myofibers with only endomysia fibronectin. The overall appearance of regenerated muscle was similar to the normal muscle compare except that the regenerated muscle showed more areas of fibrous connective tissue containing fibronectin.

Studies of effect of difference type of regeneration and healing muscle that was depended upon the size and location of injury used and experimental type of injury that are associated skin tears or intact skin tracked the presence of early appearance of fibronectin as early as after trauma, the role of fibronectin in this early stages may be explain the formation of primary matrix that will form cross linking bridges with fibrin present in the clot, (13).

That simultaneous increase in the sarcoplasm fibronectin statistical record in this study can be explain by the depletion of endomysia layer early after injury it was suspected that feedback control of fibronectin production and expression lead to increase in sarcoplasm fibronectin and these may be due to activated satellite cell which will be activated after injury or is due to accumulated of fibronectin from extracellular fluid as a result of attracted muscle cell permeability .Since fibronectin is one of component of base of several type of tissue the changes in it expression can be used to follow the fate of basement membrane during regeneration process in skeletal muscle,(3).

In a separate study, employing antibodies against laminin, fibronectin and type IV collagen, which are specific markers for basement membrane eventual loss of these components was seen in the degenerating myofibers (before the formation of myotubes,laminin and type IV collagen reappeared around the regenerated myotubes, further suggesting that the original basement membrane disappeared and a new basement membrane appeared around the regeneratedmyotubes ,finally, it is also possible that individual componentsrather than the whole basementmembrane changes sequentially (i.e., not simultaneously)during muscle degeneration and regeneration.The present study described changes in fibronectindistribution during skeletal muscle regeneration and its usefulness to the study of myogenesis in vivo. Because the alterations observed in the expression of fibronectin in ischemic and degenerating myofibers weresimilar to those occurring in vitro during cell transformation, the present model can be used to study cell alterations in vivo and their relationship to ischemia. 


\section{Acknowledgment:-}

I would like to express my sincere gratitude to my supervisor Prof. Dr .May. F. AL-Habib for her suggestion this work, her patience, great support generous help and continuous guidance throughout the course of study

\section{References:-}

1. Hung, C., Linn, G., Chow, Y. H., Kobayashi, A., Mittelsteadt, K., Altemeier, W. A. \& Duffield, J. S. (2013). Role of lung pericytes and resident fibroblasts in the pathogenesis of pulmonary fibrosis. American journal of respiratory and critical care medicine, 188(7), 820-830.

2. Tedesco, F. S., Dellavalle, A., Diaz-Manera, J., Messina, G., \&Cossu, G. (2010). Repairing skeletal muscle: regenerative potential of skeletal muscle stem cells. The Journal of clinical investigation, 120(1), 11.

3. Musarò, A. (2014). The basis of muscle regeneration. Advances in Biology, 2014.

4. Lehto, M., Duance, V. C., \&Restall, D. (1985). Collagen and fibronectin in a healing skeletal muscle injury. An immunohistological study of the effects of physical activity on the repair of injured gastrocnemius muscle in the rat. Bone \& Joint Journal, 67(5), 820-828.

5. Huijbregts, J., White, J. D., \& Grounds, M. D. (2001). The absence of MyoD in regenerating skeletal muscle affects the expression pattern of basement membrane, interstitial matrix and integrin molecules that is consistent with delayed myotube formation. Actahistochemica, 103(4), 379-396.

6. Gulati, A. K., Reddi, A. H., \&Zalewski, A. A. (1983). Changes in the basement membrane zone components during skeletal muscle fiber degeneration and regeneration. The Journal of cell biology, 97(4), 957-962.

7. Singh, P., Carraher, C., \&Schwarzbauer, J. E. (2010). Assembly of fibronectin extracellular matrix. Annual review of cell and developmental biology, 26, 397-419.

8. Dessau, W., Von Der Mark, H., Von Der Mark, K., \& Fischer, S. (1980). Changes in the patterns of collagens and fibronectin during limb-bud chondrogenesis. Development, 57(1), 51-60.

9. Gulati, A. K., Reddi, A. H., \&Zalewski, A. A. (1982). Distribution of fibronectin in normal and regenerating skeletal muscle. The Anatomical Record, 204(3), 175-183.

10. To, W. S., \&Midwood, K. S. (2011). Plasma and cellular fibronectin: distinct and independent functions during tissue repair. Fibrogenesis\& tissue repair, 4(1), 21.

11. Sottile, J., \& Hocking, D. C. (2002). Fibronectin polymerization regulates the composition and stability of extracellular matrix fibrils and cell-matrix adhesions. Molecular biology of the cell, 13(10), 3546-3559.

12. Sottile, J., Hocking, D. C., \&Swiatek, P. J. (1998). Fibronectin matrix assembly enhances adhesion-dependent cell growth. Journal of cell science, 111(19), 2933-2943.

13. Lovering, R. M., \& De Deyne, P. G. (2004). Contractile function, sarcolemma integrity, and the loss of dystrophin after skeletal muscle eccentric contraction-induced injury. American Journal of Physiology-Cell Physiology, 286(2), C230-C238. 